\title{
LA POLÍTICA SOCIAL EN LA CONSTRUCCIÓN DEL DESARROLLO HUMANO Y SOCIAL
}

José Guadalupe Vargas Hernández

\section{Resumen}

Este trabajo tiene por objetivo explorar, analizar y abundar el tema de la política social y su significancia para el desarrollo social. Al hacerlo, inevitablemente también considera las relaciones e implicaciones de la política social con el Estado, las estrategias de atención a la pobreza, los procesos de la globalización económica, la participación ciudadana, las metrópolis y el desarrollo urbano y sus implicaciones en la política ambiental.

Palabras clave: Desarrollo Social; Estado de Bienestar; Nueva Política Social; Política Social; Teorías Críticas.

\begin{abstract}
This paper seeks to analyze the meaning of social policy for social development. It takes into account the relationships and implications of social policy to the State as well as the strategies to combat poverty, the economic globalization processes, citizen participation, the metrópolis and urban development and their implications for environmental policy.
\end{abstract}

Keywords: Social Development; Welfare State; New Social Policy; Social Policy; Critical Theories.

\section{Introducción}

Este trabajo está diseñado para actuar como un foro abierto para el debate y el análisis de los temas contemporáneos centrados en las variables señaladas de esta línea de investigación, basada en las contribuciones desde diferentes acercamientos disciplinarios y con referencia contextual a la situación geográfica del desarrollo social local. Lo anterior da lugar y espacio a una diversidad de puntos de vista sobre el asunto de las diferentes implicaciones de la política social en la actual sociedad, de tal forma que también estimulan el intercambio y el debate de ideas, conclusiones, hallazgos y propuestas de cada una de las contribuciones. El objetivo fundamental explícito de las políticas sociales es la mejora de las condiciones de vida de los individuos en una sociedad.

Por lo tanto, con el noble propósito de analizar los avances de las investigaciones sobre la política social, es un paso importante para estimular el debate en un asunto tan controvertido. Con en esto en mente, la investigación materializa una contribución importante a la generación del conocimiento científico, a la creación de modelos teórico-metodológicos para 
el análisis y a la comunicación y difusión de los principales hallazgos en el contexto del Estado y la sociedad civil actual.

Este análisis se presenta de tal forma que reúna aportaciones de los académicos e investigadores juntos para compartir los productos de sus investigaciones y sus ideas sobre el tema. Su propósito es presentar un conjunto de reflexiones que reflejan algunos de los nuevos desarrollos, el pensamiento y acercamientos contemporáneos relacionados con el tópico de su investigación dentro del marco de referencia de los procesos de globalización contextualizados en lo local, desde sus diferentes antecedentes e intereses disciplinarios y científicos. Los procesos de globalización económica se articulan con los procesos políticos y sociales para caracterizar nuevas formas de organización social y la reestructuración de las relaciones sociales que cambian los modelos de integración y exclusión, marginalidad y pobreza en las sociedades contemporáneas.

Los trabajos académicos se despliegan para ofrecer desde los grandes acercamientos teórico-metodológicos así como las investigaciones empíricas. Para poner en su exacta dimensión las importantes contribuciones de los procesos de cambio de la política social en la construcción del desarrollo social es conveniente hacer algunas anotaciones teórico-metodológicas a la investigación de las políticas sociales.

\section{Reflexiones teórico-metodológicas a la investigación de las políticas sociales}

El papel de la política social se relaciona con el desarrollo económico, social y político. La búsqueda de la coordinación y coherencia de las políticas debe tomar en cuenta el hecho de que las ideas acerca de la política social y su papel en el desarrollo han cambiado con el tiempo, señalando la dificultad para encontrar claridad en los acercamientos a la inversión social, el alivio de la pobreza y la equidad. El resultado de la implementación de las políticas sociales que han transitado por cambios profundos en su diseño, es alarmante en términos de profundas desigualdades sociales y del bienestar de los individuos en la sociedad.

Las respuestas definitivas al aprovisionamiento social, pobreza y desigualdad son probables que continúen siendo elusivas, y como tales, es importante sostener las oportunidades para el debate, la experimentación, innovación y aprendizaje en los acercamientos considerados al desarrollo social. Encontrar las formas para preservar estas condiciones así como para alentar las apreciaciones profundas por los factores contextuales que dan forma a los resultados del desarrollo, pueden ser contribuciones importantes, a pesar de ser en sí mismas contra-intuitivas de las nuevas formas de coherencia y cooperación internacional.

La naturaleza de la política social como tema es por tanto relevante, no solo para los científicos y académicos de la política, la sociología y la administración pública, sino también para los estudiosos y practicantes en las 
instituciones y organizaciones de los diferentes gobiernos, negocios y organizaciones no gubernamentales y de la sociedad civil en general. A pesar de que la política social y su impacto en la sociedad han estado sujetos a un gran debate, con frecuencia de una naturaleza crítica, las evidencias continúan señalando que una mejor formulación, diseño y ejecución de una adecuada política social tiene un impacto positivo en el desarrollo social, fundamentalmente.

La primera razón para querer involucrarse en el debate de la política social en periodo de transición, tiene que ser una respuesta a los clamores hechos por el futuro del bienestar por los pensadores sociales. No obstante, al hacer un recuento analítico de los numerosos puntos de vista sobre las políticas sociales que han sido expresadas en las publicaciones científicas, tales como el Journal Social Policy, se concluye que tienden a reflejar la posición de los contribuyentes y quizás los puntos de vista de algunos de los editores colectivos, pero la búsqueda por una simple perspectiva coherente sería una tarea vana.

Las variables de la incertidumbre y la ambigüedad son con frecuencia asociadas con algunos acercamientos post-clásicos de la política social que parecen pervertirla. Por otra parte, el riesgo y la evaluación del riesgo son elementos centrales al Estado de Bienestar contemporáneo como para involucrar tanto a los acercamientos críticos, como a los más ortodoxos a la política social, apuntando a las pensiones que son un buen ejemplo para ilustrar los elementos de exclusión y las nociones de riesgo basados en los seguros y sus limitaciones inherentes para mucho de los esquemas de diseño de políticas sociales. Pero más que alguna contradicción esencial, se argumenta que es necesario un diálogo más informado y soberbio, para asegurar un intercambio franco de ideas sobre algunas clarificaciones iniciales de los principales temas de la política social.

Las estructuras políticas de los Estados nacionales enfrentan como mayor reto político-social el diseño de instituciones para la formulación e implementación de una política social que promueva el crecimiento económico y el desarrollo social bajo condiciones de restricciones y exigencias fiscales que limitan los márgenes de maniobra. El diseño institucional del Estado de Bienestar apoya la implementación de una política social para atenuar los excesos del capitalismo liberal. El diseño del Estado Benefactor, como también se le conoce, es una construcción ideológica, política y social que se sustenta en la acción del ejercicio del poder del Estado los procesos de formulación e instrumentación de las políticas sociales.

Hay terreno fértil para considerar a los Estados de Bienestar y a la política social como esencialmente modernos y diametralmente opuestos a todas las cosas postmodernas. A pesar de que hay profundos huecos en muchos de los recuentos de bienestar usados por los observadores postclásicos y un rechazo frustrante para tratar la política social con la seriedad que claman para su propio trabajo como en el caso de los acercamientos postmodernos, hay aspectos que son compatibles con las críticas al 
acercamiento de las políticas sociales críticas. Si se acepta lo que se está estableciendo en la agenda, quien quiera involucrarse con académicos familiares con los detalles de las políticas sociales desde estas perspectivas, es posible que acepte su recuento estrecho de los desarrollos del bienestar y reconozca los puntos que elevan acerca de la necesidad de repensar fundamentalmente todo.

En todo caso, las críticas del bienestar público tienden a tomar dos formas. Primero, los ingenieros sociales del Estado de Bienestar lo tomaron equivocadamente, y segundo, los actuales defensores del Estado de Bienestar continúan tomándolo equivocadamente. Si lo anterior es cierto, entonces la posibilidad de encontrar un terreno común con la política social crítica parece ser difícil.

La política social crítica define su propia posición como opuesta a la derecha radical y consciente de las inadecuaciones de los modelos Fabián (CHIARA y DI VIRGILIO, 2009) y de otros modelos ortodoxos. No siempre ha sido fácil distinguir la posición de la derecha radical, para decirlo así, de la izquierda radical en algunos temas de la política social, por lo cual hay sospechas cuando aparecen publicaciones de trabajos libertarios cuidadosamente logrados, como por ejemplo la discriminación de las drogas o la industria sexual. La gran fuerza de la política social crítica ha sido su diversidad y la amplitud de su crítica capaz de analizar las artificiales capas de diferentes formas de opresión, desigualdad y desventajas bajo una suerte de acercamiento que tiene clase, género, edad, discapacidad, etc., superpuestos unos de los otros para exigir diferentes formas de bienestar.

Los estudiosos de la política social pueden ser avisados simplemente para olvidarse de Foucault (1994, 2007a y 2007b), Braudillard (1974) y del post-clasicismo (CHIARA y DI VIRGILIO, 2009) y el postmodernismo (CHIARA y DI VIRGILIO, 2009). Si se está aterrizado en el empirismo de la política social, por alguna evidencia que los fundadores del Estado de Bienestar han sustentado en cualquier período que se pueda escoger, se mantienen ideas que no fueron consideradas benignas, incluso a los ojos de sus contemporáneos, al igual que en los tiempos actuales.

Un mal entendimiento del por qué y cómo la política social desarrollada no es asunto de detalle fino, es la tradicional preocupación del recuento de la política social de la persona con sus propios hechos contra los intereses de los sociólogos y politólogos en el amplio sentido de eventos o teoría, lo cual no es decir que existe la verdad en la que todos puedan estar de acuerdo. La historia de la política social es tan contestada como cualquier otra. Pero si el reclamo es que el desarrollo del bienestar ilustra las limitaciones del ejercicio modernista, puede ser de más ayuda reconocer las diversas y diferentes formas que el bienestar ha tomado. Además, la historia y desarrollo de la política social actualmente ilumina la modernidad y revela mayor complejidad y ambigüedad de lo que es con frecuencia pensado.

La ciudadanía puede no llegar a ser una gran idea para la política social sin el reconocimiento de los cambios en la localidad en la cual han ocurrido 
desde 1945. Se necesita recordar el más viejo significado del término, esto es el status de habitante de un pueblo o de una gran ciudad. Lo que está siendo más claro es que las nuevas desigualdades emergen desde 1940, lo cual tiene que ser tomado en cuenta cuando se discute el objetivo de la política social. Una perspectiva crítica distintiva en el papel del Estado de Bienestar es el vínculo estrecho entre las políticas sociales y económicas en el período de la posguerra y la acumulación y funciones de legitimación atribuidas a la política social. Una de las más poderosas ideas que informan la dominante tradición de la política social y la administración pública hasta antes de los setentas fue la noción de que las políticas sociales se preocupaban por producir integración social.

Los analistas de la política social se han movido fuera del concepto de pobreza hacia el concepto de exclusión social desde los inicios de los noventas y no es una coincidencia que se corresponda con el empeoramiento del clima económico, con más altos perfiles sociales y demográficos de las necesidades y mayores presiones fiscales sobre la provisión del bienestar. Si el nuevo enfoque de la política social ha sido promotor de valores y responsabilidad personal, las instituciones sociales fuera del gobierno deben de ser más movilizadas.

Ciertos supuestos subrayan el pensamiento de la política social en este tiempo, tales como un compromiso al empleo pleno, las relaciones entre los gobiernos del centro y los locales para la provisión de bienestar, las aportaciones de las organizaciones filantrópicas y de caridad, la participación de los ciudadanos y las organizaciones de la sociedad civil, etc. La forma en las cuales las desigualdades afectan las vidas de mucha gente es a través de la consolidación de áreas de políticas públicas las cuales no son convencionalmente pensadas como política social.

La denominada globalización de los principios de la administración pública y las prácticas de política social son parte de un proceso de globalización más amplio, causado económicamente por un cambio de un modelo de un mundo de economías nacionales distintas a una economía global en la cual la producción es internacionalizada y el capital financiero fluye libremente e instantáneamente entre los países. Estos procesos son motivados por la revolución de la información que ha ayudado a debilitar las barreras nacionales de tal forma que, incluso los marcos de referencia de las políticas sociales que afectan a los ciudadanos individuales están llegando a ser más sensibles a las influencias internacionales.

El estudio académico de la política social y la administración pública ha respondido a los enormes cambios económicos y sociales: estudios de desempleo, nuevas formas de pobreza, el impacto regional de la declinación económica, el estatus de alteridad de las mujeres, el impacto de las desventajas económicas y sociales en las minorías étnicas, etc. La economía y la sociedad han cambiado y también el campo de la política social. En la respuesta de los gobiernos a los inextricables procesos de globalización económica, se ha dado nueva forma a un amplio rango de políticas sociales, 
las cuales tienen por objeto tratar no solamente de aliviar la pobreza material sino también otros fenómenos sociales disfuncionales tales como el crimen, la adicción a las drogas, la emergencia de la familia con padre soltero, y el tema de cómo los valores son generados y pasados a los hijos, entre muchos otros asuntos.

La mayoría de los países en Latinoamérica están ahora en camino hacia las economías totalmente competitivas y están en una posición de diseñar y financiar sus políticas sociales. Los procesos de ajuste estructural del Estado recomendados por los organismos internacionales para la inserción del modelo económico neoliberal, implicaron cambios estratégicos en las estructuras económicas para afianzar la economía de libre mercado. A su vez, la implementación de esta nueva política económica neoliberal sustenta una nueva política social con fuertes consecuencias en las estructuras sociales y el bienestar de la sociedad.

Los cambios en el diseño de la nueva política social que se está implementando en los últimos tres decenios dan respuesta al contexto de los procesos de globalización económica sustentados en propuestas teóricasideológicas de menos Estado asistencial y más mercado de Milton Friedman (BEYER, 1995) de la Escuela de Chicago. La política social en Latinoamérica ha tenido cambios profundos en los últimos tres decenios orientados por la reducción de la responsabilidad y participación del Estado y la consecuente expansión de las actividades del mercado en los procesos de formulación e implementación, financiamiento, provisión, administración y transferencia de servicios sociales y asistenciales.

El modelo de Estado neoliberal ha despojado el diseño de la política social como una responsabilidad del Estado de Bienestar para intervenir en la procuración del bienestar de la sociedad, transfiriendo esta responsabilidad al mercado, ideológicamente considerado como el mejor agente en la asignación de los recursos para la solución de los problemas sociales. Sin embargo, el uso de métodos de shock de las reformas del mercado ha traído a la luz problemas extremadamente agudos, no solamente en la esfera económica sino también en la esfera social en las condiciones de actividades de la población entera.

Por otra parte, se sugiere que la política social necesita ser más reflexiva, más diversa y más sensitiva a las teorías sociales que si bien no ofrecen soluciones del todo, si algunos acercamientos a los problemas sociales contemporáneos. Además, muchas de las preocupaciones de la política social crítica están siendo ahora compartidas por los denominados comentaristas ortodoxos de las políticas sociales que han sido en sí mismos más críticas por algún tiempo.

Esto también puede de alguna forma explicar la negligencia del análisis post-clásico dentro de los círculos académicos y científicos de la política social. El punto clave aquí, es que el escepticismo de los recuentos post-clásicos asegura un punto de vista crítico de la política social. Ya sea que aquellos quienes se consideran a sí mismos como parte de un acercamiento de las 
políticas sociales críticas aceptan a los post-clásicos como compañeros viajeros es debatible, pero claramente hay solamente un pequeño paso entre ser escéptico y ser crítico acerca de los clamores de los ingenieros sociales. En algunos sentidos, al menos cualquier analista relacionado con la política social, se ha vuelto crítico y puede quizás ser clasificado como parte de una creciente crítica de la modernidad. Así, puede pensarse que la política social en los albores del siglo XXI encarna el ejercicio modernista y por tanto debe ser condenado.

Una estrategia integral de bienestar social llama por una efectiva coordinación y complementación en el diseño, implementación y evaluación de las políticas sociales. La orientación social de las políticas sociales presupone la elección de las prioridades sociales, la estimulación con propósito de ciertas esferas y direcciones de las actividades, la producción de bienes de consumo y servicios, el apoyo por la esfera social, etc., y la implementación de programas sociales.

Las políticas sociales ya no pueden estar limitadas a los servicios asistenciales caracterizados por el Estado de Bienestar sin referencia a las interconexiones entre estos servicios y las otras áreas de las políticas públicas, a la dominante concepción de ciudadanía dentro de las políticas sociales y la administración pública. El problema para el individuo es construir un sentido auténtico de identidad en la recreación de un contexto de soporte en un ámbito público que adecua la ciudadanía social en contraposición a la ciudadanía global centrada solamente en el consumo, como un concepto integrador para las políticas sociales.

Una de las más poderosas ideas que informan la dominante tradición de la política social y la administración pública fue la noción de que las políticas sociales se preocupaban por producir integración social. La estabilidad de la comunidad en la cual las personas viven tiene un importante tratamiento de las percepciones del futuro, $y$ en particular de las implicaciones futuras de las políticas sociales. Sin embargo, está ahora muy pronunciada la tendencia en el campo de la investigación de las políticas sociales que empieza a debilitar la credibilidad de las estadísticas de las políticas sociales y de los aspectos de la investigación orientada por las políticas sociales en sí mismas.

Estos son algunos aspectos del debate reciente sobre las relaciones de las políticas sociales, cuyo tratamiento inadecuado solo refleja escepticismo y crítica desde dentro de las políticas sociales, a pesar de algunas serias observaciones relacionadas con el tratamiento de los análisis que son significativos para las políticas sociales críticas. Desafortunadamente, la tradición del escepticismo y la crítica dentro de la política social no ha sido reconocida por los post-clásicos como por ejemplo, los postmodernistas. Por lo tanto la crítica al postmodernismo ofrecida no está en contradicción a la política social, pero la definición del Estado de Bienestar y de política social es estrecha y las historias que las informan son peculiares porque en otros 
respectos son consideradas como categorías unitarias y comprensivas por lo que son rechazadas.

La estrecha definición de bienestar que los analistas han usado es vista como que no ayuda y no guía, por lo que rehusar a reconocer las críticas de bienestar que han durado y que sin embargo, poco contribuyen a las políticas sociales críticas es irritantemente miope. El debate ha sugerido que algunos aspectos del análisis de las políticas sociales son importantes para entender los temas vitalmente importantes en el entendimiento del bienestar contemporáneo.

Sin embargo, inicialmente puede ser una tentación para responder. Las políticas sociales sean ortodoxas o críticas, están ciertamente en un período transicional. La cuestión crucial es que el diálogo vale la pena y se sugiere que es porque cualquier intento debe ser para ir más allá de los programas de política social diseñados e implementados por gobiernos de izquierda y de derecha, lo cual requiere ser informado por una apreciación propia de las críticas que han precedido. La izquierda acepta con una fe consistentemente crítica, tanto en la estrategia y la filosofía normativa que informó del acercamiento ortodoxo a la política social. Sin embargo, la tradición socialista sobre el Estado Bienestar confronta una formidable lista de problemas, lo cual pone en duda si el acercamiento a las áreas sustantivas de la política social es correcto.

Además, se sugiere que el criticismo relacionado con cualquier posibilidad de la ingeniería social, debe también ser informado por una política social crítica que ha sido siempre escéptica. Si hay un sentido general de escepticismo, un sentimiento de que las políticas sociales han fallado o la creencia de que hay poco que pueda hacerse para resolver las divisiones sociales, es crucialmente importante ser claro acerca de las razones para tales análisis.

El punto serio que emerge de este sumario de los debates es que la fe en los formuladores, diseñadores y ejecutores de las políticas sociales se ha sacudido fuertemente. Los acercamientos tradicionales a las políticas sociales se perciben cercanos a los políticos, muchas de las veces sin que se establezcan algún tipo de diálogo con una amplia población constituyente. A pesar de que hay profundos huecos en muchos de los recuentos de bienestar usados por los observadores con enfoque tradicional y un rechazo frustrante para tratar la política social con la seriedad que claman para su propio trabajo, hay aspectos del acercamiento.

En este sentido, lo tratado abre posibilidades de análisis considerando la pertinencia del tópico al contexto local, lo cual abre las puertas para involucrarse con académicos más familiares con los detalles de las políticas sociales. Esto hace posible aceptar su recuento de los desarrollos del bienestar y reconocer los puntos que se elevan acerca de la necesidad de repensar fundamentalmente contenido y forma de la política social. Además, se muestran las evidencias considerables de que el proceso de reflexión y 
debate sobre el futuro del bienestar y las políticas sociales ha estado y seguirá estando bajo revisión por algún tiempo.

Más allá de ser uniforme, el campo de la política social está compuesto de muchos sub-campos, a pesar de que frecuentemente se encuentran dentro del mismo sistema de bienestar algunos programas de política social. Cada área de política social, de salud, educación, seguridad social, etc., no solo despliega una estructura diferente sino que activan muy diferentes habitus y pueden involucrar diferentes intereses, a pesar de que dentro del contexto se presente una estructura similar de dominación. Por lo tanto, la interface de las diferentes agencias de política social y beneficiarios también adquieren un carácter muy diferente con programas de políticas sociales universales debido a los factores que influencian el esfuerzo del bienestar, los mecanismos involucrados, las fuerzas de trabajo que han sido desarrollados dentro, etc., lo cual determina por qué los programas de política social son más desarrollados en algunos países que en otros y de una región a otra.

En cada estadio y en cada espacio, la política social incluyendo las leyes que benefician a los pobres, es crucial en la cambiante lucha de clases, con el miedo del desorden y la ingobernabilidad de la movilización popular, por lo cual estos se constituyen en los antecedentes y consecuencias de los sistemas a la administración del desarrollo social. Desde esta perspectiva, la tendencia de los análisis de la política social considera que debe ser el sistema de la administración del desarrollo social crecientemente realizado a través de los órganos locales. Estos mismos órganos locales deben ser una expansión de la autorregulación y la creación de condiciones para el auto desarrollo de sujetos de administración.

Consecuentemente se trata de un mecanismo económico en el cual las influencias de control económico están orientadas directamente hacia las condiciones de desarrollo social, no necesariamente por métodos de influencia directa sino también por indirecta y a través del discurso. En esta conexión, la determinación del aspecto subjetivo-sustantivo de la política social, las condiciones de su formulación e implementación y la delimitación de poderes de los sujetos de la administración pública de diferentes jerarquías están también en el debate de las políticas sociales. Por lo tanto, la política social local debe ser una actividad integrada basada en las metas federales y suplementadas por las metas regionales.

El impacto mentiroso de las políticas dañinas y estadísticas controladas por los gobiernos, junto a la privatización de la pobreza a través de las políticas gubernamentales directas, también parecen haber tenido un incremento de la interferencia política en la construcción de la agenda de investigación de la política social. Se sugiere que se necesita promover el diálogo, pero no es simplemente un caso de política social para escuchar a los teóricos sociales. Por lo tanto, hace bien hacer una reflexión y poner un poco más de atención a los debates desde dentro de la política social, estimulados con la sugerencia de que las políticas sociales pueden ser compatibles con los modelos de desarrollo imperantes. 
Sin embargo, el diálogo y el debate sobre las políticas públicas necesita ser promovido y no puede haber tal cosa como la política social que rechaza escuchar otras voces críticas ni pueden los post-clásicos continuar descuidando una política social que en todo el espectro político ha sido crítica y escéptica. Es importante hacer notar que a la fecha los comentaristas críticos en la política social han estado más preparados para considerar los trabajos sobre los análisis post-clásico de las políticas sociales que viceversa. Por una parte, esto es frustrante ya que el estudiante de las políticas sociales puede también fácilmente llegar a ser involucrado en un debate sociológico rarificado que se aparta de los temas sustantivos que los relacionan.

Hasta ahora muchos de los que se identifican con la política social crítica pueden negarla entusiastamente a pesar de que algunos quieran emplear el reduccionismo peculiar. Peculiar, porque descansa no tan fácilmente con un modelo teórico. Reduccionismo porque la idea de ciertas funciones claves es difícil de sostener a la luz de varias críticas. Pero dejando esas cuestiones de un lado, hay más profundos aspectos que provocan disturbios en los recuentos de la política social.

Sin embargo, el Estado Benefactor entra en contradicciones ideológicas con el liberalismo ortodoxo primero, y más tarde con la ideología neoliberal de libre mercado que rechaza la intervención del Estado en la política social. Se considera como una debilidad del diseño de Estado Benefactor, que no considera la dinámica de los mercados y flujos financieros que inciden en la producción de bienes y servicios y la emergencia de crisis financieras. Esta debilidad es la principal causa de que la ideología neoliberal acuse de inviabilidad del Estado Benefactor a favor de un cambio de estrategia de política social.

El nuevo diseño neoliberal de política social modifica las relaciones entre el Estado y la sociedad para orientarla a grupos específicos en situaciones de extrema miseria. En todo caso la responsabilidad del Estado de una política social universal queda limitada a la provisión de servicios sociales y transferencias focalizadas a grupos específicos en extrema pobreza.

Si se busca un campo común de análisis de las políticas sociales, es importante notar que un elemento de los acercamientos críticos a la política social es que han sido consistentemente escépticos de aquellos quienes han clamado que el Estado de Bienestar y el Estado mínimo de corte neoliberal están promoviendo la buena sociedad. Cuestionan para quien es buena y el bien para qué. Han sido el tema de muchas contribuciones a la política social crítica que cuestionan las medidas de bienestar que parecen reforzar formas particulares de la familia, roles de género, productivismo, dependencia y prejuicios étnicos y raciales. Las contribuciones a la política social crítica han sido consistentemente escéptica de la burocracia del bienestar público y las prácticas que fallan en el aliento a involucrarse con la experiencia, necesidades y puntos de vista de los grupos constituyentes a quienes están dirigidos nominalmente. 
Si los analistas post-clásicos de las políticas sociales consideran que éstas son más que estrechamente o inadecuadamente operantes, esto no detracta de las implicaciones de sus críticas por una política social crítica. En algunos aspectos el borrarse de las fronteras y las posiciones epitomiza las incertidumbres asociadas con la critica a los análisis post-clásicos en donde las tropas de varios ejércitos ideológicos saltan las trincheras en la tierra de nadie, llena de cuerpos y de minas pero donde dejan una política social crítica. Primeramente hay un tema inicial sobre si la perspectiva de la política social crítica existe en un sentido diferente que como una norma de alegar, las publicaciones están vivas y dan cuenta de que hay una política claramente definida y una noción de lo que es la política social crítica aunque sea debatible.

No resulta tan fácil apuntar a una política social ortodoxa no intentada por los eventos de las pasadas décadas. Hay quienes consideran el asunto de la política social como foro, e incluso aliado que trata de todas las preocupaciones, además los temas de identidad, diferencias, agencia y diversidad, todos ahora cercanamente asociados con la iglesia de los enfoques post-clásicos y de la postmodernidad, los cuales han sido más debatidos por algún tiempo dentro de los círculos de las políticas sociales. Se puede argumentar que la política social crítica está de hecho articulada en muchas de las preocupaciones asociadas con la postmodernidad y para un tiempo considerable, hay y parece ser una prima facie como caso para sugerir que hay algunas medidas de acuerdos entre algunos analistas de la postmodernidad, algunos recuentos de la política social crítica y un creciente acercamiento crítico ortodoxo a la política social.

Sin embargo, hay aspectos del análisis post tradicional que requieren mayor atención. Con el argumento que en tanto la política social crítica, como los acercamientos ortodoxos, se han movido más cerca de las identidades de los ciudadanos, de tal forma que aquellos campos han llegado a borrase. Por tanto, se sugiere que tratar de definir dos campos dentro de la política social puede ser de ninguna ayuda e infructuoso. Un recuento ortodoxo puede llegar a ser más crítico y una tarea difícil que confronta quien quiera tomar un punto de vista en defender las políticas sociales, es como hacer esto sin atraer a los grupos de ciudadanos tradicionales pero crecientemente fragmentados.

\section{Lo evidente e inevitable: los procesos de cambio de la política social en la construcción del desarrollo social}

Algunos de los cuestionamientos abiertos que se hacen a estos actores responsables de la formulación e implementación de las políticas sociales, son los siguientes:

$\checkmark$ ¿Cuáles son las tendencias de los procesos de cambio de la política social en la construcción del desarrollo social? 
$\checkmark$ ¿Cuáles son las expectativas de los diferentes agentes de cambio responsables de cambio de la política social en la construcción del desarrollo social?

$\checkmark$ ¿Cuáles son las expectativas del ser humano para volver a ser un sujeto de cambio social histórico de la política social?

$\checkmark$ ¿Cuáles son las condiciones del entorno económico, político y social que faciliten la instrumentación de una política social que tienda a niveles de desarrollo humano más elevados mediante la eliminación de las injusticias y desigualdades sociales al mismo tiempo que garantice el ejercicio pleno de la libertad individual?

Los procesos de globalización económica pueden crear las condiciones suficientes para la promoción del cambio de la política social orientada a la construcción del desarrollo humano y social. La globalización como un fenómeno económico, político y social constituye un espacio por explorar para una revaloración conceptual del desarrollo humano, en donde el ser humano vuelva a ser un sujeto de cambio social. Este espacio se identifica con una cultura global centrada en los derechos humanos. Para lograrlo, estos procesos de globalización económica tienen que modificar los principios económicos que actualmente le dan sustento, centrados en el cambio estructural que impulsa las grandes tendencias centrífugas afectando los mecanismos de cohesión social y política.

Los procesos de globalización económica traen consigo adecuaciones del sistema político-social, el ajuste de nuevos arreglos de las instituciones y sistemas de gobernanza, la formación de una cultura de paz, el ejercicio de una ética global, la cohesión de las relaciones sociales por normas, la promoción del desarrollo sustentable, etc.

Los logros y alcances de procesos de globalización económico profundizados por un modelo de desarrollo económico neoliberal desde los años setentas del siglo pasado, permiten hacer un replanteamiento de las responsabilidades institucionales del Estado en materia de políticas sociales y motivan a los académicos e investigadores al estudio de nuevos objetos evidentes en los cambios de paradigmas.

La nueva política social emerge de una ideología neoliberal que estrecha la acción del Estado capitalista en materia de asistencia y bienestar social solo facultado para operar los cambios sociales que las estructuras económicas dominantes demandan para beneficio de sus propios intereses y en perjuicio de la solución de las demandas de la sociedad en su conjunto. Un Estado con esta configuración capitalista neoliberal polariza la distribución de la riqueza con efectos disfuncionales que profundizan la pobreza, la marginalización y la exclusión social. Un Estado con esta configuración de capitalismo neoliberal por más democrático que intente ser, sus contradicciones internas impiden la promoción de igualdad de oportunidades, capacidades y derechos de los ciudadanos. 
Finalmente, apuntando al acercamiento de los procesos de cambio de la política social en la construcción del desarrollo social, la responsabilidad recae en los agentes capaces de promover estos cambios, tales como los académicos, investigadores y analistas, la sociedad civil, las tecnologías de la información y comunicación (TICs) empleados por los medios, los sujetos individuales titulares de los derechos derivados, etc.

Primeramente, si los estudiosos son alentados para reflejar las políticas sociales en una moda crítica, intentando hacer fácil imaginar y entender el contenido de los diferentes temas centrados en la política social, no ha habido muchas oportunidades para los analistas de políticas sociales para estar cercanamente involucrándose en enmarcar las políticas. Sobre todo en los pasados 30 años, durante los cuales se han puesto presumiblemente responsables en un rango de reformas al infinito en tanto las leyes progresan para dar operatividad al modelo de desarrollo económico.

Virtualmente, cada quien en el mundo académico de la política social tiene que ser crítico de las políticas del gobierno. Por lo tanto se puede argumentar que ya no hay distintivamente una política social crítica a pesar de que sería una exageración sugerir que la política social crítica está en peligro de evaporar en el seco calor del cinismo del análisis post-clásico.

Hay evidencias considerables de que el proceso de reflexión y debate sobre el futuro del bienestar y las políticas sociales ha estado bajo revisión y cambio por algún tiempo. Dada la duración y la intensidad de los debates dentro de la política social sobre el significado e intenciones del Estado parece remarcable que nadie debería hacer estos supuestos. Mas importante es, sin duda alguna, argumentar que han sido las luchas de aquellos que confían en el bienestar público las que han hecho mucho para generar una política social más reflexiva pero distinta. Son estas voces analíticas críticas más que los tradicionales grupos de constituyentes de estos nuevos sujetos de identidades a los que se debe poner más atención.

Parece aparente, por lo tanto, que hay mucho que explorar e investigar, y todavía, muchas maneras de entender esas exploraciones e investigaciones. El campo de la investigación relacionado con la política social es un campo abierto a otras disciplinas académicas, que si bien no es nuevo, está en pleno desarrollo. La combinación del trabajo de investigación y análisis de todas estas contribuciones, mejora y refuerza el intercambio y discusión de las ideas y hallazgos desde la amplitud intelectual que ofrecen los campos de varias disciplinas, más que ofrecer un punto de vista estrecho desde el enfoque de cualquier disciplina en particular.

En los años recientes, la sociedad civil está jugando un papel protagónico en la promoción de un cambio cualitativo en el desarrollo social a través de las exigencias de mejores programas de política social suministrado y administrado por un marco institucional en el que confluyen procesos de cooperación entre el Estado, el mercado y la misma sociedad civil. El esquema estratégico de la nueva política social se centra en principios de autogestión y sentido de corresponsabilidad social y política de los actores particulares 
individuales facilitados por procesos de gestión pública y con el soporte de organizaciones sociales, organismos no gubernamentales o la sociedad civil en general. Esta tendencia requiere de grandes acuerdos para dar direccionamiento a los cambios cualitativos en los procesos productivos dimensionados por las nuevas formas de la existencia y desarrollo humano.

La participación activa de la sociedad civil animada para la extensión de los beneficios de programas de política social a todos los individuos, sobre todo cuando se constituye en factor de cambio social, permite lograr el equilibrio entre las fuerzas del libre mercado y la responsabilidad del Estado a intervenir, entre el ejercicio de la libertad individual y la justicia social, entre la eficiencia del mercado y el intervencionismo del Estado para una distribución equitativa del ingreso y para atenuar los efectos nocivos de la economía capitalista.

El involucramiento de los individuos sujetos de los beneficios de las políticas sociales hace sumamente relevante la promoción de procesos de participación ciudadana como un requerimiento fundamental de la corresponsabilidad con las instituciones del Estado y el mercado para el diseño, formulación e implementación de políticas orientada a la promoción y mejoramiento del bienestar de la sociedad.

El uso pertinente de las tecnologías de información y comunicación (TICs) son instrumentos poderosos para habilitar las capacidades humanas que faciliten los cambios de las políticas sociales más propensas al desarrollo humano y social, de tal forma que permitan la expresión de los individuos como sujetos históricos transformadores de su propia realidad económica, política y social. Desde esta perspectiva, el sistema capitalista considerará el esfuerzo transformador del ser humano no necesariamente como un medio de producción sujeto como cualquier otra mercancía al intercambio comercial en el mercado laboral, sino como un fin en sí mismo para alcanzar los niveles más elevados de desarrollo humano.

A pesar de que todos los actores y agentes reconoce la importancia de profundizar las reformas de política social en la que todos están de acuerdo, sin embargo se presentan diferentes posiciones con respecto a la dirección que debe darse a estas reformas. Se concluye que los procesos de cambio de las políticas sociales son evidentes e inevitables para la construcción del desarrollo humano y social.

\footnotetext{
José Guadalupe Vargas Hernández es Maestro en Administración de Empresas con especialidad en Dirección Industrial por Pacific States University, Doctor en Economía por Keele University, England y Doctor en Administración Pública por Columbia University. En la actualidad es Profesor investigador del Centro Universitario de Ciencias Económico Administrativas de la Universidad de Guadalajara.

E-mail: jvargas2006@gmail.com
} 


\section{Referencias}

BEYER, Harald. Selección de escritos políticos y económicos de Milton Friedman. Estudios Públicos, Santiago, n. 60, p. 431-484, primavera 1995.

BRAUDILLARD, Jean. Crítica de la economía política del signo. Cidade do México: Siglo XXI, 1974.

CHIARA, Magdalena; DI VIRGILIO, María Mercedes. Gestion de la politica social Conceptos y herramientas. Buenos Aires: Prometeo Editorial, 2009. (Colección Temas Urbanos - coedición con la UNGS).

FOUCAULT, Michel. Curso del 14 de Enero de 1976. In: FOUCAULT, Michel. Microfísica del poder. Madrid: Las ediciones de la Piqueta, 1994. p. 139-152.

. La gubernamentalidad. In: GIORGI, Gabriel; RODRÍGUEZ, Fernín (Comps.). Ensayos sobre Biopolítica, excesos de vida. Barcelona: Paidós, 2007a. p. 187-215.

- La vida: la experiencia y la ciencia. In: GIORGI, Gabriel; RODRÍGUEZ, Fernín (Comps.). Ensayos sobre Biopolítica, excesos de vida. Barcelona: Paidós, 2007b. p. 187-215.

Texto recebido em 19/01/2012.

Aprovado em 06/04/2012. 